\title{
Maintenance olaparib for BRCA-mutated ovarian cancer (OC) patients in first-line and platinum-sensitive relapsed (PSR) settings: maximizing treatment opportunities
}

\author{
Andres M Poveda, ${ }^{1}$ Alfred Sackeyfio, ${ }^{2}$ Michael Friedlander ${ }^{3}$ \\ 'Department of Gynecologic Oncology, Initia Oncology, Valencia, Spain; ${ }^{2}$ AstraZeneca, Cambridge, UK; \\ University of New South Wales Clinical School, Prince of Wales Hospital, Randwick, Australia
}

Poster no. IGCS19-0574

\section{Introduction}

- Olaparib (Lynparza ${ }^{\circledR}$ ) is approved for maintenance treatment in women with advanced BRCA-mutated (BRCAm) OC who have achieved a complete or partial response (CR/PR) following completion of first-line platinum-based chemotherapy (PBC). It is also approved for maintenance treatment in PSR OC, irrespective of BRCA mutation status.

- In Phase III randomized trials, maintenance therapy with olaparib demonstrated an efficacy benefit versus placebo in patients with BRCAm advanced OC who had a CR/PR to PBC in the newly diagnosed (SOLO-1; NCT01844986) 1 and PSR OC (SOLO-2 /ENGOT-Ov21; NCT01874353) 2 settings.

- Based on the SOLO-1 study results, olaparib maintenance therapy for women with newly diagnosed BRCAm advanced OC also provides an apparent enduring treatment benefit, which is important in preventing or delaying platinum resistance and in reducing or delaying the burden of disease and adverse events associated with multiple lines of PBC in relapsed settings.

- The direct cost burden of OC after first-line therapy has been reported to escalate for women with PSR $\mathrm{OC}$ as a result of second-line treatment costs, hospitalizations and the management of treatmentrelated adverse events. ${ }^{3}$

- Cost analysis of maintenance therapy with olaparib in women with newly diagnosed BRCAm advanced $\mathrm{OC}$ has found that the cost of maintenance therapy stabilizes after 2 years and reduces to a low level over the remainder of the patient's lifetime. ${ }^{4}$

- Moreover, the costs for women with newly diagnosed BRCAm advanced OC who do not receive olaparib maintenance following first-line PBC, regardless of disease progression status, increase after 2 years because of second or later-line treatment. ${ }^{4}$

- Here, we investigate missed opportunities for olaparib maintenance therapy in the setting of newly diagnosed OC.

\section{Methods}

\section{Data identification}

- A targeted literature review was conducted to obtain response rates to $\mathrm{PBC}$ in newly diagnosed patients with advanced BRCAm OC and those with BRCAm PSR OC.

- The search yielded few results in either cohort. The use of an adjustment factor was considered.

\section{Newly diagnosed BRCAm OC}

- Searching relevant manuscripts did not reveal PBC response rates applicable in the newly diagnosed setting

- GOG 218 study authors previously predicted response rates to PBC to be $75 \%{ }^{5}$ in a newly diagnosed nonbiomarker-specific population. As this response rate was obtained from a non-biomarker-specific population, a BRCA response rate adjustment factor was applied.

\section{BRCAm PSR OC}

Searching relevant manuscripts identified a $64.6 \%$ response rate to $\mathrm{PBC}$ in BRCAm patients in the secondline setting published by Alsop et al. ${ }^{6}$

- Platinum sensitivity data for second-line PBC was determined from the proportion of patients in the placebo arm of the SOLO-1 trial who were progression free after 6 months (80.6\%).

\section{BRCA response rate factor}

- To determine the BRCAm population response rates to PBC, a BRCA mutation response rate factor was derived from two studies that assessed PSR OC patient populations:

- Aghajanian et $a^{8}$ reported response rates in non-biomarker patients as $57.4 \%$

Alsop et $a{ }^{6}$ determined response rates in BRCAm patients as $64.6 \%$

The BRCA mutation response rate factor was calculated as (BRCA mutation response rate [Alsop 64.6\%])/(nonbiomarker response rate [Aghajanian 57.4\%]) $=1.125$

It was assumed that the numerical relationship between the BRCA mutation and non-biomarker response rates in the PSR OC population would apply to the newly diagnosed population.

\section{Missed opportunity for olaparib treatment calculation}

- The proportion of newly diagnosed BRCAm patients who are eligible for olaparib and who would experience possible resistance to, and potential failure of, second-line PBC was calculated by ([proportion of newly diagnosed BRCAm patients eligible for olaparib - proportion of BRCAm PSR OC patients eligible for olaparib]/[proportion of newly diagnosed BRCAm patients eligible for olaparib]) $\times 100 \%$.

\section{Results}

- In a hypothetical cohort of 1000 BRCAm patients, 844 (84\%; Figure 1) will be eligible for maintenance olaparib following first-line PBC, and 439 (44\%; Figure 2) will be eligible for olaparib in the PSR OC setting (Table 1).

- Based on this, $48 \%$ ([844-439]/844) of patients who were eligible for olaparib in the newly diagnosed setting may not receive olaparib maintenance therapy in the second-line setting.

Table 1. Estimation of proportion of patients with BRCAm OC who have achieved a complete or partial response to PBC and are eligible for olaparib maintenance therapy in both the newly diagnosed OC and PSR OC settings

\begin{tabular}{|c|c|c|c|}
\hline Patient setting & Cohort, $n$ & Calculation & BRCAm population eligible for olaparib, $n(\%)$ \\
\hline $\begin{array}{l}\text { Newly } \\
\text { diagnosed } \\
\text { BRCAm OC }\end{array}$ & 1000 & $\begin{array}{c}1000 \\
\times 0.75 \text { (first-line response rate) } \\
\times 1.125 \text { (BRCA response rate factor) }\end{array}$ & $844(84)$ \\
\hline $\begin{array}{l}\text { BRCAm PSR } \\
\text { OC }\end{array}$ & 1000 & $\begin{array}{c}1000 \\
\times 0.75 \text { (first-line response rate) } \\
\times 1.125 \text { (BRCA response rate factor) } \\
\text { x } 0.806 \text { (platinum sensitivity) } \\
\text { x } 0.646 \text { (second-line response rate) }\end{array}$ & $439(44)$ \\
\hline
\end{tabular}

Figure 1. Proportion of BRCAm patients eligible for olaparib following first-line PBC Patients with

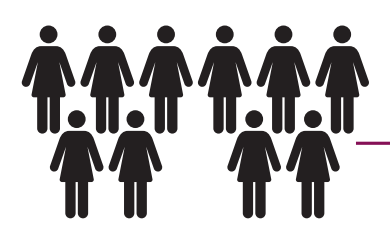
First-line platinum chemotherapy $\underset{\sim 75 \% \text { response rate }(\mathrm{CR} / \mathrm{PR})^{5}}{\stackrel{\sim 125}{\mathrm{BRCA} \text { response rate factor }}}$ Proportion of patients eligible
for olaparib with newly for olaparib with newly Mimil Figure 2. Proportion of BRCAm PSR OC patients eligible for olaparib Patients with Proportion of patients eligible advanced OC for olaparib with newly

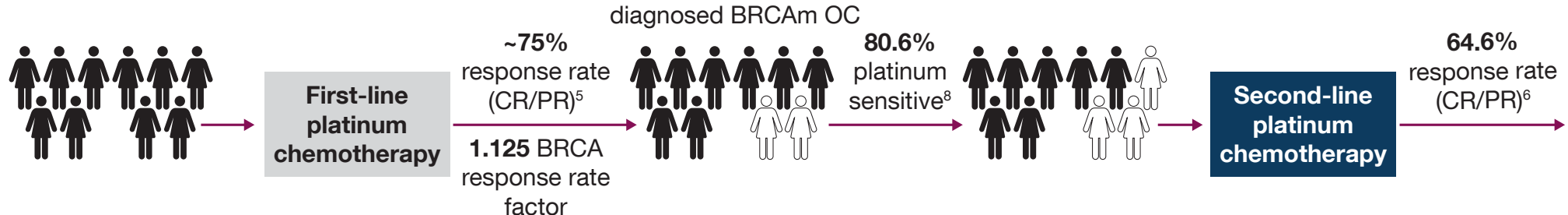
Proportion of patients eligible for olaparib

\section{Conclusions}

- These analyses demonstrate that an additional $48 \%$ of BRCAm OC patients may obtain the treatment benefit from olaparib demonstrated in the SOLO-1 study if treated following first-line PBC rather than in a PSR setting

- Potential advantages of olaparib maintenance therapy following first-line PBC may include:

- Prevention or delay of relapse or resistance and extension of the time until subsequent treatment is required Reduction in the adverse events, poor quality of life, disease burden and direct costs associated with progressive disease.

- A limitation of this analysis was not identifying published response rates for newly diagnosed BRCAm patients; a derived BRCA response rate factor was used to determine those response rates.

\section{References}

1. Moore Ket al. N Engl J Med 2018;379:2495-505

2. Pujade-Lauraine E et al. Lancet Oncol 2017;18:1274-84.

3. Gordon LG et al. Int J Gynecol Cancer 2010;20:757-65.

4. AstraZeneca. Data on file. SOLO-1 budget impact model. 2019

5. Burger RA et al. N Engl J Med 2011;365:2473-83.

6. Alsop K et al. J Clin Oncol 2012;30:2654-63.

7. AstraZeneca. Data on file. SOLO-1 clinical study report. 2018

8. Aghajanian C et al. J Clin Oncol 2012;30:2039-45.

\section{Acknowledgments}

This study was funded by AstraZeneca and is part of an alliance between AstraZeneca and Merck Sharp \& Dohme Corp., a subsidiary of Merck \& Co., Inc., Kenilworth, NJ, USA (MSD). Medical writing assistance was provided by Helene Wellington from AMICULUM Ltd, funded by AstraZeneca and MSD. 